Please do not remove this page

RMIT

UNIVERSITY

\title{
Compositional relaxation on the approach to the glass transition in a model trehalose solution
}

Hannam, Stephen; Daivis, Peter; Bryant, Gary

https://researchrepository.rmit.edu.au/esploro/outputs/9921858886601341/filesAndLinks?institution=61RMIT_INST\&index=null

Hannam, S., Daivis, P., \& Bryant, G. (2019). Compositional relaxation on the approach to the glass transition in a model trehalose solution. Physical Review E, 99(3), 1-12.

https://doi.org/10.1103/PhysRevE.99.032602

Document Version: Accepted Manuscript

Published Version: https://doi.org/10.1103/PhysRevE.99.032602

Repository homepage: https://researchrepository.rmit.edu.au

(c) 2019 American Physical Society

Downloaded On 2023/04/26 20:34:05 +1000

Please do not remove this page 


\title{
The study of compositional relaxation on the approach to the glass transition in a model trehalose solution
}

\author{
Stephen D. W. Hannam, Peter J. Daivis, ${ }^{*}$ and Gary Bryant \\ School of Science and Centre for Molecular and Nanoscale Physics, \\ RMIT University, GPO Box 2476, Melbourne Vic. 3001, Australia.
}

(Dated: June 11, 2021)

\begin{abstract}
Molecular dynamics simulation was used to study the temperature dependence of the mutual diffusion coefficient $D_{m}$ and the intermediate scattering function of equilibrium and metastable aqueous solutions of the cryoprotectant molecule trehalose at very low (2.2 and 9 wt.\%) and very high (80 and 95 wt.\%) concentrations. The simulations were conducted over a range of temperatures approaching the glass transition temperature $T_{g}$ for each concentration. Similar to a recent observation made on a glass forming model polydisperse colloidal suspension [Hannam et. al., Phys. Rev. E, 2017, 96 022609], we confirmed by a set of independent computations that $D_{m}$ is responsible for the long-time decay of the intermediate scattering function. We observed that $D_{m}$ decreased on the approach to the glass transition temperature, resulting in an extremely slow long-time decay in the intermediate scattering function that culminated in the arrest of compositional fluctuations and a plateau in the intermediate scattering function at $T_{g}$. In both cases, crystallisation requires a change in the composition of the solution, which is a process controlled by $D_{m}$. This transport coefficient can either increase or decrease as solidification is approached, because it depends on a product of thermodynamic and mobility factors. Our observations show that in both cases, for the glass forming liquids, it is observed to decrease, while for a previously studied monodisperse colloidal suspension which crystallises easily, it increases. The similarity in the behaviour of these two very different glass forming systems (the polydisperse colloidal suspension and the sugar solution) shows the importance of the mutual diffusion coefficient to our understanding of vitrification and suggests a possible distinction between between glass forming and crystallising solutions.
\end{abstract}

\footnotetext{
* Electronic mail: peter.daivis@rmit.edu.au
} 


\section{INTRODUCTION}

The cryopreservation of biological cells and tissues is essential to many diverse applications, such as artificial insemination, organ transplantation, virus research [1] and the preservation of endangered species [2]. However, if ice crystals form inside cells during the cooling process, this usually leads to cell death [3]. Vitrification is a cryopreservation process in which the sample is preserved in a glassy state, and is one of the means by which cellular damage can be avoided [4]. Vitrification requires high concentrations of molecules that facilitate formation of the glassy state, and sufficiently fast cooling rates that crystallization can be avoided [5].

In nature this is achieved by the accumulation of high concentrations of disaccharides (sucrose and trehalose), which occur in significant quantities in certain plants, seeds, and invertebrates adapted to endure desiccation and freezing $[6,7]$. The success of small sugars as cryoprotectants has two major facets: first, they reduce dehydration damage to cell membranes, through mechanisms which have been investigated at length [8-13]; and second, they encourage vitrification, which is the focus of this work.

In nature these molecules are created inside cells. However, they are not suitable on their own as artificial cryprotectants as they are not membrane-permeating, and cannot penetrate cells. In cryopreservation, it is common to use permeating cryoprotectants such as DMSO, which can encourage vitrification and hinder ice formation, but high concentrations of such cryoprotectants are chemically toxic, disrupting membranes and damaging cells. This can be mitigated somewhat by combining glass forming sugars with lower concentrations of permeating cryoprotectants to achieve successful cryopreservation or desiccation tolerance $[14,15]$.

This study focusses on trehalose, which has the highest glass transition temperature of any of the disaccharides $[6,9,10]$, though the physical mechanisms underlying this are far from clear. It is a nonreducing disaccharide that results from the combination of two Dglucopyranose molecules through $(1 \rightarrow 1)$ glycosidic linkages. The $\alpha, \alpha$-trehalose dimer is shown in Fig. 1. The propensity for solutions of trehalose to vitrify has been attributed by some workers to its ability to alter the water structure, making it less ordered and thus hindering ice formation [11-13]. It has also been suggested that the vitrification properties are closely related to its ability to affect the dynamics of the water $[9,12]$. 


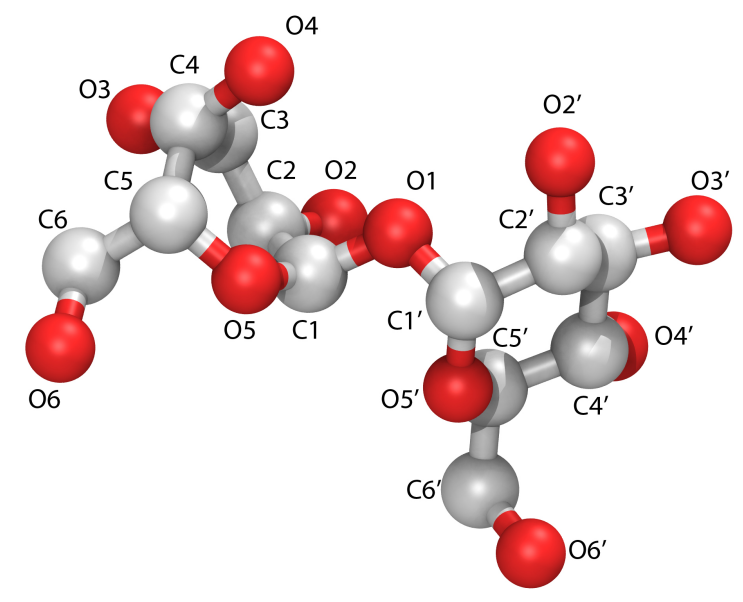

FIG. 1. $\alpha, \alpha$-Trehalose where hydrogen atoms have been omitted for clarity. All carbon and oxygen atoms have been labeled.

In studies of the change in dynamics of the solution in the lead up to vitrification, great attention is paid to single particle dynamics, often through study of the self diffusion coefficient [16]. Though this property does provide useful information on the decrease in mobility in the approach to the glass transition, our previous work on colloidal suspensions [17, 18] has highlighted the importance of the collective dynamics, as seen through the interdiffusion coefficients, in determining whether a system will form a glass. That work showed that the glass transition in bimodal colloidal dispersions is correlated with a decrease in a particular interdiffusion coefficient $D_{-}$to a negligible value, resulting in a plateau forming in the intermediate scattering function that signifies the formation of a glass.

This $D_{-}$coefficient is an eigenvalue of the $2 \times 2$ matrix of interdiffusion coefficients for the ternary colloidal fluid, and is directly related to the system's ability to make compositional changes. Since compositional changes are often necessary for a quenched multicomponent system to meet the local stoichiometry for crystallization, it was hypothesised that in this case, the inability to make compositional changes was the cause of the observed crystallization inhibition, which ultimately led to the formation of a long-lived metastable glassy state.

The viscosity and self diffusion coefficient both decrease as the concentration of a binary solution increases or its temperature decreases, regardless of its glass forming ability. In other words, the mobility of the solute decreases as the freezing point is approached via a concentration increase or a temperature decrease for both crystalizing and vitrifying solu- 
tions. However, this is not necessarily the case for the mutual diffusion coefficient, which is a product of a mobility and a thermodynamic factor. This can be seen, for example in our results for the concentration dependence of the mutual diffusion coefficient of a model unimodal (monodisperse) colloidal dispersion [17]. When polydispersity is introduced, it can have a dramatic effect. In the bimodal colloidal dispersion that we previously studied, we found that the diffusion coefficient governing changes in composition at fixed total concentration decreased dramatically to nearly zero for a glass forming system. Strong inhibition of crystallization can also be observed in binary systems when the size ratio of the two components is in the appropriate range. In this case, the mutual diffusion coefficient is correspondingly seen to decrease as the concentration is increased [19].

There has recently been strong interest in the role of compositional changes in controlling the rate of crystallization. Nandi et al. [20] studied the effects of alloy composition on crystallization. They found that in some binary alloys the glass forming ability of the alloy depended strongly on composition, and that in some cases at least, the glass forming ability increased when the entropy penalty for demixing of the alloy in the interfacial region was maximised. Demixing is a collective process governed by both kinetic and thermodynamic factors, similar to the mutual diffusion coefficient that we study here.

Pedersen et al. [21] have recently found that the well-known Kobb-Andersen model of a glass forming alloy composed of $80 \%$ large A particles and 20\% smaller B particles actually crystallizes after a sufficiently long time into an FCC crystal of pure A particles in coexistence with a solution of A plus B. Again, the inhibition of crystallization is sensitive to the composition in such a way that general slowing down of the dynamics is not sufficient to explain the optimisation of the glass-forming ability of the alloy. A property such as the mutual diffusion coefficient, which incorporates both thermodynamic and frictional factors may provide useful information on the complex interplay between composition, frictional slowing and glass forming ability.

A phase-field modelling study of compositional relaxation dynamics for a glass forming binary atomic mixture via the intermediate scattering function published by Berry and Grant [22] also supports the view that collective, rather than single particle frictional properties are most relevant to models of caging, and crystallization inhibition. The two models featured in their work both include a parameter that accounts for the temperature dependence of the collective mobility associated with the decay of composition fluctuations. This essentially 
corresponds to the inverse of the collective friction factor appearing in the mutual diffusion coefficient.

Our aim in this work is to determine whether the behaviour observed in our previous study of bimodal colloidal fluids is more universal. In particular, we ask whether similar behaviour can be observed in a water-saccharide solution on the approach to the glass transition. Water-saccharide solutions and colloidal suspensions are both glass forming systems, but differ greatly in the sense that the latter can be considered analogous to simple atomic or hard-sphere alloys, while the former is a solution of complex non-spherical molecules. Therefore if such an analogous relationship exists, it may point to common underlying physical principles governing these two systems.

When ice crystals form and grow in a solution they exclude the solutes, and thus the concentration of the solutes increases in the unfrozen solution. This is known as the freezeconcentration effect [23]. One of the suggested cryopreservation mechanisms for trehalose is that, as the solute is excluded from the crystal, there is a local increase in the solute concentration near a growing ice interface, which inhibits ice-crystal growth [24]. The ability of the solution to demix and allow ice crystals to grow relies on its ability to make compositional changes at the interface.

In the saccharide solution, the transport coefficient that determines the relaxation of composition is the mutual diffusion coefficient $D_{m}$. It determines how quickly a concentration gradient (or concentration fluctuation) will decay. Though there have been some experimental measurements of $D_{m}$ for other saccharide solutions [25], to the authors knowledge the data for trehalose is extremely limited [26]. Therefore this will be one of the first studies of the mutual diffusion coefficient of trehlose across a broad temperature and concentration range. $D_{m}$ can also be related to the decay of the intermediate scattering function $[27,28]$, which is a key quantity measured in dynamic scattering that shows the arrest of concentration fluctuations in the approach to the glass transition. If $D_{m}$ plays the same role in vitrification in the saccharide solution as $D_{-}$does in the colloidal suspension, it would offer valuable insight into the processes involved in vitrification, and may help to explain why trehalose is such a good vitrifier compared to other disaccharides.

The outline of this paper is as follows: first we give a summary of the computational model used, and describe how we calculate the mutual diffusion coefficient in equilibrium MD using time correlation functions. Then we independently determine the glass transition 
temperature of the solution by quenching to low temperatures, and calculate the constant pressure heat capacity upon heating. We go on to examine how the mutual diffusion coefficient (and all the factors that contribute to it) behave on the approach to the previously determined glass transition temperature.

We then perform a multiexponential analysis of the water intermediate scattering function over the same range of concentrations and temperatures, to show that the long-time decay of the intermediate scattering function is governed by the mutual diffusion coefficient. We demonstrate that the extremely long relaxation time of the fluid, which manifests as an extremely slow decay, and ultimately the arrest of the intermediate scattering function, is linked to the reduction of $D_{m}$ to a negligible value. This allows a physical interpretation of the cause of the glass transition in this system, and shows a striking similarity between the underlying physics governing the mechanism of the glass transition in the saccharide solution and the colloidal suspension.

\section{THEORY}

\section{A. Intermediate Scattering Function}

The molecular intermediate scattering function $F_{\alpha \beta}(k, \tau)$ is defined as the normalised auto-correlation function of a Fourier component of the molecular number density:

$$
F_{\alpha \beta}(k, \tau)=\frac{1}{N} \frac{\left\langle n_{\alpha}(\mathbf{k}, \tau) n_{\beta}^{*}(\mathbf{k}, 0)\right\rangle}{S_{\alpha \beta}(k)}
$$

where $n_{\alpha}(\mathbf{k}, t)$ is given as

$$
n_{\alpha}(\mathbf{k}, t)=\sum_{j=1}^{N_{\alpha}} \exp \left(-i \mathbf{k} \cdot \mathbf{r}_{j}(t)\right)
$$

and $N$ is the total number of molecules, $N_{\alpha}$ is the number of molecules of species $\alpha$ and $\mathbf{r}_{j}(t)$ denotes the center of mass position of molecule of particle $j$ at time $t$. The static structure factor $S_{\alpha \beta}(k)$ is defined as:

$$
S_{\alpha \beta}(k)=\frac{1}{N}\left\langle n_{\alpha}(\mathbf{k}, 0) n_{\beta}^{*}(\mathbf{k}, 0)\right\rangle .
$$

where ${ }^{*}$ signifies the complex conjugate. In an MD simulation, the wavevector $\mathbf{k}$ being studied must be consistent with the periodic boundary conditions of the simulation box:

$$
\mathbf{k}=\frac{2 \pi}{L}\left(a_{1}, a_{2}, a_{3}\right)
$$


where $a_{i}$ is an integer and $L$ is the length of the simulation box (in this work the box is cubic so $\left.L_{x}=L_{y}=L_{z}\right)$.

From eqn (4) we see that the lowest non-zero $k$ value that can be studied in an MD simulation has a magnitude of $\left|\mathbf{k}_{\text {min }}\right|=2 \pi / L$. As the fluid is isotropic the correlation functions $F_{\alpha \beta}(k, \tau)$ and $S_{\alpha \beta}(k)$ only depend on the magnitude $k=|\mathbf{k}|$, so an average is done over all $\mathbf{k}$ of equal magnitude. The intermediate scattering functions shown in this work will all be molecular center of mass density autocorrelation function of the water molecules $F_{11}(k, \tau)$. In order to simplify the notation the subscripts will be dropped, and we will simply denote $F(k, \tau)=F_{11}(k, \tau)$. However if we need to distinguish between the two species, subscript 1 represents the water while subscript 2 represents the trehalose.

\section{B. Calculation of the Mutual Diffusion Coefficient}

The mutual diffusion coefficient can be calculated from equilibrium MD simulations using a combination of Green-Kubo [29, 30] and Kirkwood-Buff theory [31]. The diffusion coefficient for a 2-component isothermal fluid is defined by the linear flux-force relation [32]:

$$
\mathbf{J}_{1}^{0}=-D_{m} \nabla n_{1}
$$

where $\mathbf{J}_{1}^{0}$ is the diffusive molecular flux defined as

$$
\mathbf{J}_{1}^{0}=n_{1}\left(\mathbf{v}_{1}-\mathbf{v}^{0}\right)
$$

Here $\mathbf{v}_{1}$ is the center of mass velocity of the water molecules and $\mathbf{v}^{0}$ is the volume average streaming velocity given by

$$
\mathbf{v}^{0}=\sum_{\alpha=1}^{2} n_{\alpha} v_{\alpha} \mathbf{v}_{\alpha}
$$

where $v_{\alpha}$ is the partial molecular volume of species $\alpha$.

The diffusion coefficient is defined in terms of the flux measured relative to the volume average streaming velocity, but it is more convenient for computation to calculate the phenomenological coefficients defined by flux laws expressed in terms of the barycentric (mass average) streaming velocity. The relationship between the phenomenological coefficients with respect to one reference velocity and the diffusion coefficients measured relative to another can be written as [32];

$$
D_{m}=\frac{L_{11} n_{2} v_{2}}{c_{2}^{2}}\left(\frac{\partial \mu_{1}}{\partial n_{1}}\right)_{p, T, n_{2}}
$$


where $L_{11}$ is the phenomenological coefficient, $\mu_{1}$ is the chemical potential of the water, $c_{2}$ is the mass fraction of trehalose and $p$ is the pressure. Therefore to determine the mutual diffusion coefficient we need to determine the thermodynamic factor $\partial \mu_{1} / \partial n_{1}$, the partial molecular volume of the sugar $\nu_{2}$ and phenomenological coefficient $L_{11}$.

The phenomenological coefficient is calculated from Green-Kubo theory [29, 30] which states that

$$
L_{11}=\frac{V}{3 k_{B} T} \int_{0}^{\infty}\left\langle\mathbf{J}_{1}(\tau) \cdot \mathbf{J}_{1}(0)\right\rangle d \tau .
$$

where $T$ is the temperature and $\mathbf{J}_{1}$ in this instance is calculated in the barycentric reference frame of the simulation box. The thermodynamic factor and partial volumes can be calculated from Kirkwood-Buff theory [31]. The well known expression found by Kirkwood and Buff states that for a fluid with 2 species the thermodynamic factor is calculated from

$$
\frac{1}{k_{B} T}\left(\frac{\partial \mu_{1}}{\partial n_{1}}\right)_{p, T, n_{2}}=\frac{1}{n_{1}}+\frac{G_{12}-G_{11}}{1+n_{1}\left(G_{11}-G_{12}\right)}
$$

and the partial volume of species 2 from

$$
v_{2}=\frac{1+n_{1}\left(G_{11}-G_{12}\right)}{n_{1}+n_{2}+n_{1} n_{2}\left(G_{11}+G_{22}-2 G_{12}\right)} .
$$

The volume integrals of the radial distribution functions $G_{\alpha \beta}$ are calculated from

$$
G_{\alpha \beta}=\int\left(g_{\alpha \beta}(\mathbf{r})-1\right) d \mathbf{r}=4 \pi \int r^{2}\left(g_{\alpha \beta}(r)-1\right) d r
$$

where $g_{\alpha \beta}(r)$ is the radial distribution function of species $\alpha$ and $\beta$. These integrals can be difficult to calculate as statistical error in $g_{\alpha \beta}(r)$ at large $r$ is magnified by the factor of $r^{2}$, so the numerical integrals may not converge. As was shown in the previous work $[17,18]$ a much simpler way to calculate this quantity is through the partial structure factors:

$$
G_{\alpha \beta}=\frac{1}{x_{\alpha} x_{\beta} n}\left[\lim _{k \rightarrow 0} S_{\alpha \beta}(k)-x_{\alpha} \delta_{\alpha \beta}\right],
$$

where $n$ is the total molecular number density of all species. By calculating the low- $k$ values of the partial structure factors $S_{\alpha \beta}(k)$, and extrapolating to $k \rightarrow 0$, the values of $G_{\alpha \beta}$ can be calculated in a much simpler way.

\section{Multiexponential Analysis}

One goal of this work is to determine the effect that trahalose has on the decay of the intermediate scattering function, to determine whether the long-time decay can be associated 
with the mutual diffusion coefficients. We do this via a multiexponential analysis technique similar to our previous work for the colloidal system [17, 18], which is ultimately based on the arguments of Barocchi and coauthors [33]. They showed that the complete behavior of any normalized autocorrelation function of a classical many-body system can be described by a generalized Langevin equation, the exact solution of which can be written as an infinite sum of exponential functions

$$
C(t)=\sum_{j=1}^{\infty} A_{j} \exp \left(z_{j} t\right)
$$

where $A_{j}$ and $z_{j}$ are mode amplitudes and decay coefficients, respectively. Such modes can be associated with relaxation channels in the system. If $A_{j}$ and $z_{j}$ are complex quantities, the corresponding mode and its complex conjugate are both present in the series and, taken together, they represent an exponentially damped oscillation. Otherwise, real $A_{j}$ and $z_{j}$ define a purely exponential decay. An approximate solution to the generalized Langevin equation can be found by truncating eqn (14) at a finite number of terms. The behaviour of the coefficients can then be studied by fitting the resulting function to experimental and simulation data. This procedure can be difficult, due to the large number of free fitting parameters. Barocchi and coauthors found that the number of free fitting parameters can be reduced by constraining the solution. They showed that the zero time properties of the solution given in eqn (14) must obey the relation

$$
\left[\frac{d^{m} C(t)}{d t^{m}}\right]_{t=0}=0
$$

where $m$ is an odd integer. When a finite number of exponential terms are retained, eqns (14) and (15) can only be valid for $m$ up to a certain value depending on the approximation level and the model assumed. The combination of eqns (14) and (15) allows the number of free fitting parameters to be reduced.

The minimum number of terms that need to be retained in eqn (14) to accurately fit the intermediate scattering function data depends on the state point being studied. However we found that for the pure water system at the wavevectors and temperatures we studied, three real and one complex mode was needed to model the complex decay of the intermediate scattering function. The addition of the trehalose molecules causes an additional real exponential decay mode to emerge which decays orders of magnitude slower than any of the decay modes observed in the pure water system. We will endeavour to show that this 
additional slow decay mode is associated with relaxation of composition and has a decay rate proportional to the mutual diffusion coefficient.

\section{SIMULATION METHODS}

In this work we have chosen to model the trehalose molecule using the OPLS potential [34] which was developed for liquid systems and has been further optimized for all-atom simulations of carbohydrate solutions [35]. It is a fully flexible, all-atom model which includes charges, Lennard-Jones (LJ) interactions as well as linear bond length, bond angle and dihedral potentials. The parameters for a bending interaction between an sp3 carbonether oxygen-acetal carbon were used to approximate the bending at the glycosidic linkage. A detailed description of the implementation of the OPLS force field is given elsewhere [36]. In this work we use the SPC/E potential [37] for the water molecules in the solution. This model is rigid and unpolarisable but still captures many of the correct properties of real water. It has been shown to reproduce the glass transition temperature better than other non-polarisable water models [38], however it does not accurately reproduce details of the equilibrium phase diagram of water. Clearly it would be best to use a model which reproduces all of these properties. However, such a model does not currently exist, so the SPC/E model is used here as it best reproduces the glass transition, which is the focus of this study.

All of the models used in this work include LJ and coulombic interactions. The trehalose molecule has bonded interactions with spring potentials, while the bonds on the water molecule are held fixed using the SHAKE algorithm. The LJ parameters were chosen from the references above [35]; the cutoff distance in this work was set to $12 \AA$ for all atoms. The usual Lorentz-Berthelet combining rules were used to calculate cross-interaction parameters. A long-range correction was applied to the energy and virial to account for the truncation of the potential at the cutoff. The long-range Coulombic interactions were calculated using the Ewald summation method.

All simulations were run using the MD package LAMMPS [39] and results were postprocessed using in-house code. Simulations at each packing fraction were done under NPT conditions at a range of temperatures but always at a constant pressure of 1 atmosphere. The time integration scheme used follows the time-reversible measure-preserving Verlet integrator

derived by Tuckerman et al. [40] with a time step of 1 fs. The temperature is held fixed 
using a Nosé-Hoover thermostat while the pressure is held fixed using a Nosé-Hoover type barostat, both with a damping parameter of 100 fs. The number of water and trehalose molecules in each simulation is shown in Table. I.

TABLE I. Weight $\%$ concentration of trehalose, number of water molecules $N_{W}$, number of trehalose molecules $N_{T}$ and glass transition temperature found $T_{g}$.

\begin{tabular}{llll}
\hline Weight & $N_{W}$ & $N_{T}$ & $T_{g}(K)$ \\
$\%$ & & & \\
\hline 0 & 1728 & 0 & 180 \\
2.2 & 1728 & 2 & 181 \\
9.0 & 1728 & 9 & 180 \\
80.0 & 256 & 64 & 255 \\
95.0 & 64 & 64 & 306 \\
\hline
\end{tabular}

At each concentration we attempted to study systems at temperatures close to the glass transition temperature $T_{g}$. However, the ability to do this was limited at higher concentrations as the trehalose molecules were not completely soluble at certain temperatures, resulting in a solution that was inhomogeneous. At higher trehalose concentrations, analysis of the structure factor $S(k)$ showed the solution to be inhomogeneous, possibly due to aggregation of the solute. Only results for homogeneous systems are discussed in this paper. The method for determining $T_{g}$ will be shown next.

\section{RESULTS}

\section{A. Glass Transition Temperature}

In order to study the dynamics on the approach to the glass transition, we must first determine the glass transition temperature for this model at each concentration. We determined the glass transition temperature by following a technique that mimics the procedure used in differential scanning calorimetry (DSC) experiments. It exploits the fact that the glass transition is indicated by an inflection in the heat capacity upon heating from a glass $[38,41,42]$. The procedure was as follows: systems were equilibrated at temperatures well 
above the glass transition temperature (300 K for 0 wt.\%, 2.2 wt.\% and 9 wt.\% solutions, and $500 \mathrm{~K}$ for $80 \mathrm{wt} . \%$ and $95 \mathrm{wt} \% \%$ solutions), then the reference temperature of the thermostat was decreased linearly in time to cool the solution. A cooling rate of $3 \times 10^{10} \mathrm{~K} / \mathrm{s}$ was used to quench the systems from the initial temperature down to $100 \mathrm{~K}$. This was followed by heating back to the initial temperature at the same rate with the total enthalpy being calculated and saved every 1 ps. This was repeated 25 times and the results were averaged over all runs to improve sampling statistics. Then, we used Matlab to apply a smoothing spline fit to the averaged enthalpy data with a smoothing parameter of $10^{-6}$ in order to conduct numerical differentiation. The constant pressure heat capacity $C_{p}$ was calculated by numerical differentiation of the total enthalpy versus temperature. The values for $C_{p}$ as a function of temperature are shown in Fig. 2 for each concentration studied. The glass transition temperature was determined by fitting the linear regions before and after the initial increase in $C_{p}$, then taking $T_{g}$ as the intercept as is customary [42].

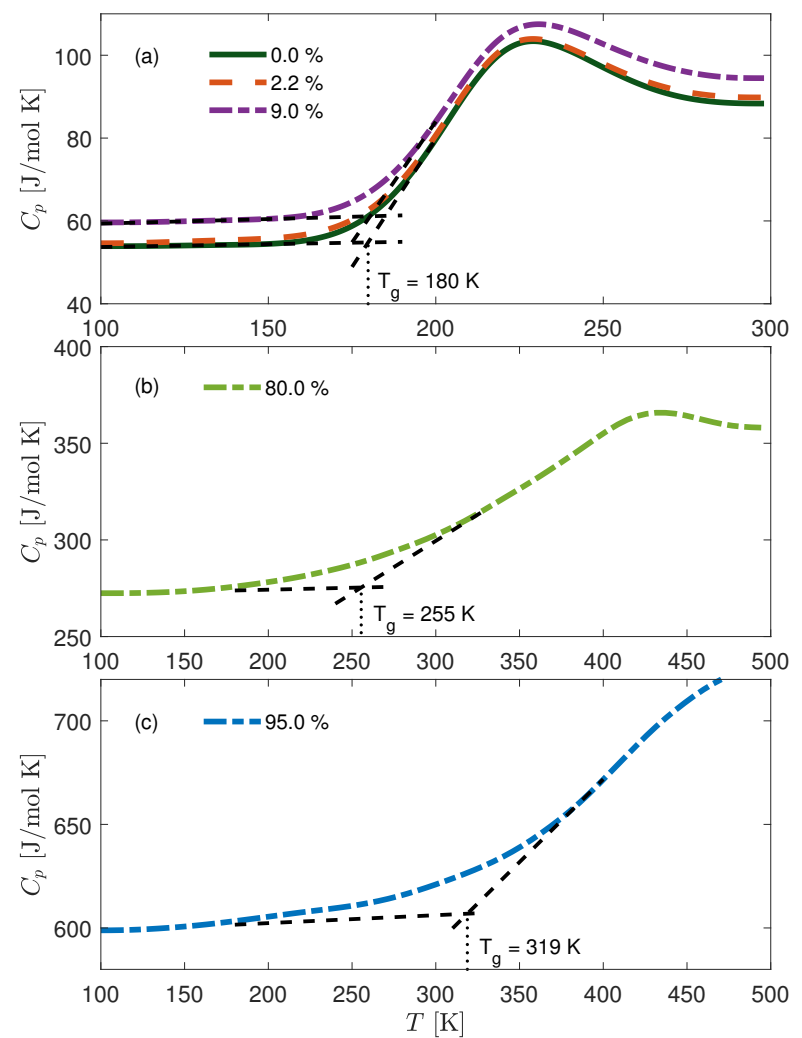

FIG. 2. Plot of the constant pressure heat capacity $C_{p}$ as a function of temperature for trehalose wt.\% concentrations shown in the legend. 
For pure water, the glass transition temperature $T_{g}$ was found to be at $\approx 180 \mathrm{~K}$. This is slightly lower than the value of $T_{g}$ calculated by Kreck and Mancera for the same cooling rate of $\approx 188 \mathrm{~K}$ [38]. There is a slight arbitrariness as to which range of the data to fit which could account for the difference. Kreck and Mancera also neglected long-range corrections to the LJ potential that could also slightly shift the glass transition temperature. A value of $180-188 \mathrm{~K}$ is above the experimental value of $T_{g}$ for pure water, which has been estimated in differential scanning calorimetry (DSC) measurements of hyperquenched water to be $136 \mathrm{~K}$. This was found by detecting the small increase in the heat capacity of water associated with vitrification $[43,44]$, though some interpret this as a prepeak preceding the true glass transition, which has been theorized to occur at the higher temperature of $165 \mathrm{~K}$ [43-45]. However this can not be tested as this falls within water's 'no mans land' where crystallisation occurs too quickly for the supercooled fluid to be studied. Determining $T_{g}$ from MD simulation usually results in a higher value than is found experimentally as $T_{g}$ is highly dependent on the cooling rate, which is orders of magnitudes faster in MD than in experiments.

The inclusion of trehalose increases the glass transition temperature, thus favouring vitrification, though not in a linear fashion. The inclusion of small concentrations of trehalose does not significantly increase the glass transition temperature. The addition of $2.2 \mathrm{wt} . \%$ and 9 wt.\% only increases the temperature by a couple of degrees, making it difficult to resolve since the value of $T_{g}$ has significant uncertainty. In line with what is observed in experiment, a large concentration of trehalose is required to raise the glass transition significantly [46].

This is observed in the higher concentration solutions shown in Fig. 2b and 2c, where at 80 wt.\% the glass transition temperature is estimated to be $255 \mathrm{~K}$, very close to the experimental value of $\approx 252 \mathrm{~K}$ [46]. A further increase to $95 \mathrm{wt} . \%$ results in a glass transition of $311 \mathrm{~K}$ compared to the experimental value of $\approx 327 \mathrm{~K}[46]$. Therefore, at 95 wt.\% concentration the solution would now be a glass under ambient conditions. $T_{g}$ is difficult to determine at higher concentration because the width of the temperature range over which the glass transition occurs was found to grow. This makes the heat capacity $C_{p}$ transition less sharp, and so the extrapolation from the two linear regimes is more uncertain. 


\section{B. Mutual Diffusion Coefficients}

In order to calculate accurate values for the mutual diffusion coefficient from eqn (8), accurate values of the phenomenological coefficient $L_{11}$, partial molecular volume of trehalose $v_{2}$ and the thermodynamic factor $\partial \mu_{1} / \partial n_{1}$ are needed. The phenomenological coefficient $L_{11}$ was calculated from the integral of the water molecule mass-flux correlation functions defined in eqn (9). The mass-flux correlation functions were calculated at intervals of 5 fs out to a maximum delay time of 100 ps for the low concentrations solutions $(<10$ wt.\%) and out to $1 \mathrm{~ns}$ for the high concentration solutions. This small interval of 5 fs allows for a high resolution when calculating the integral of the max flux correlation function and means any short time dynamics that may occur is captured, while the maximum delay time is chosen so that any long time correlations have decayed away and the calculated integral will converge. Numerical integration with the trapezoid rule was performed on the correlation functions and all the integrals were found to converge within the maximum delay time. The resulting values of the phenomenological coefficients are shown in Fig. 3 for (a) 2.2 wt.\% solution and (b) 95 wt. $\%$ solutions.

The $L_{11}$ coefficient is the proportionality constant that describes how much number density flux would result from a given chemical potential gradient. As we might have expected, the value of $L_{11}$ is observed to decrease as the temperature decreases. The lowest temperature at which this quantity was calculated at each concentration was slightly above the corresponding glass transition temperature. Attempts to calculate $L_{11}$ at lower temperatures than $T_{g}$ result in a value indistinguishable from zero, showing that the particle movement is arrested. The dependence of $L_{11}$ on temperature is similar at both concentrations shown in Fig. 3. This trend is observed at all concentrations studied, though there are difficulties calculating this quantity at intermediate concentrations as the sugar is supersaturated for a larger range of temperatures above the glass transition [46]. As we will see later, it is the $L_{11}$ coefficient that dominates the dependence of the mutual diffusion coefficients $D_{m}$ on temperature for this system, rather than the thermodynamic factor or partial molecular volume.

To determine the thermodynamic factor $\partial \mu_{1} / \partial n_{1}$ and partial molecular volumes $\nu_{\alpha}$, values

for the integrals of the radial distribution functions $G_{\alpha \beta}$ are needed. The $G_{\alpha \beta}$ were calculated from the zero- $k$ values of the partial static structure factors $S_{\alpha \beta}$ using eqn (13). As an 

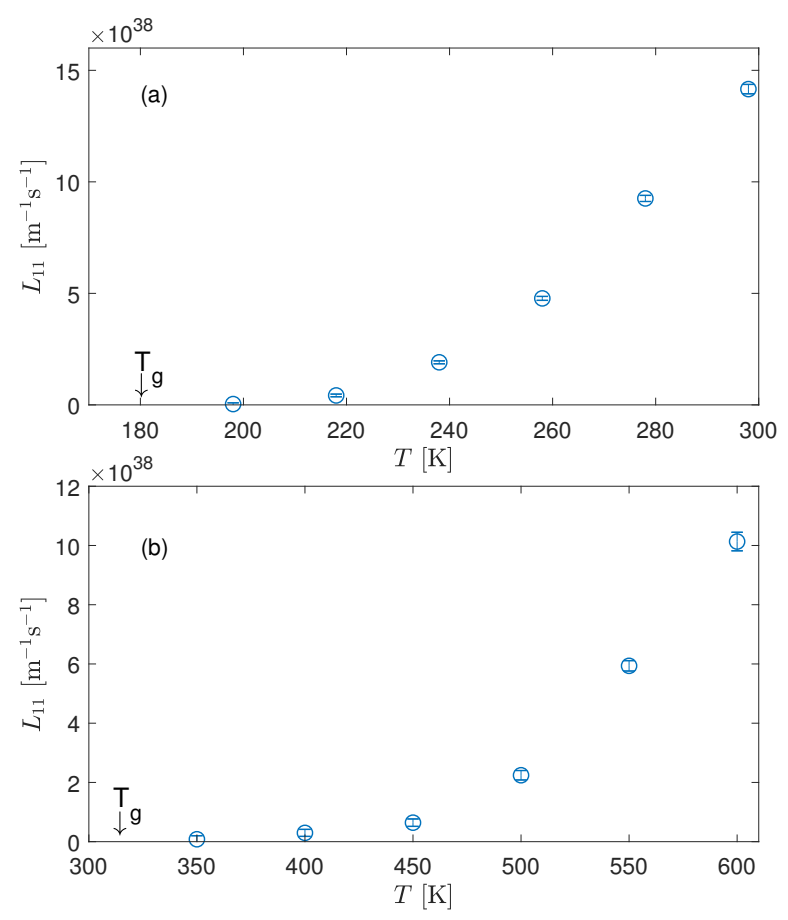

FIG. 3. Plot of the phenomenological coefficient $L_{11}$ calculated from eqn (9) for (a) 2.2 wt.\% and (b) 95 wt.\% trehalose for temperatures approaching each concentrations corresponding glass transition temperature $T_{g}$. Note the x-axis scales differ.

example of how the $S_{\alpha \beta}(k \rightarrow 0)$ were calculated, we have shown data for the concentration of 95 wt.\% and temperature of $600 \mathrm{~K}$ in Fig. 4 . These functions are plotted against $k^{2}$ as $S_{\alpha \beta}(k)$ is found to be even in $k$. Although it is possible that $S(k)$ could be a nonanalytic function of $k$, and could therefore also depend on odd or fractional powers of $|k|$, we saw no evidence of this in our data. Therefore to determine $S_{\alpha \beta}(k \rightarrow 0)$, a fourth-order polynomial in $k^{2}$ was fitted to $S_{11}$ (water-water), $S_{12}$ (water-trehalose) and $S_{22}$ (trehalose-trehalose) data and extrapolated back to $k=0$. At lower concentrations, a lower order polynomial was used if it was found to fit the data well. For the low concentration systems ( $<10 \mathrm{wt} . \%)$, the $S_{22}$ was usually quite noisy and did not have a strong dependence on $k$ due to the small number of trehalose molecules. Therefore, in order to determine $S_{22}(0)$ in these systems a simple average was taken over all the $k$ values studied.

This analysis was done for all concentrations and temperatures studied. Then, all values calculated for $S_{\alpha \beta}(k \rightarrow 0)$ were used in eqn (13) to calculate $G_{\alpha \beta}$. The values for $G_{\alpha \beta}$ were used in eqn (10) to calculate the thermodynamic factor $\partial \mu_{1} / \partial n_{1}$ and eqn (11) to calculate 

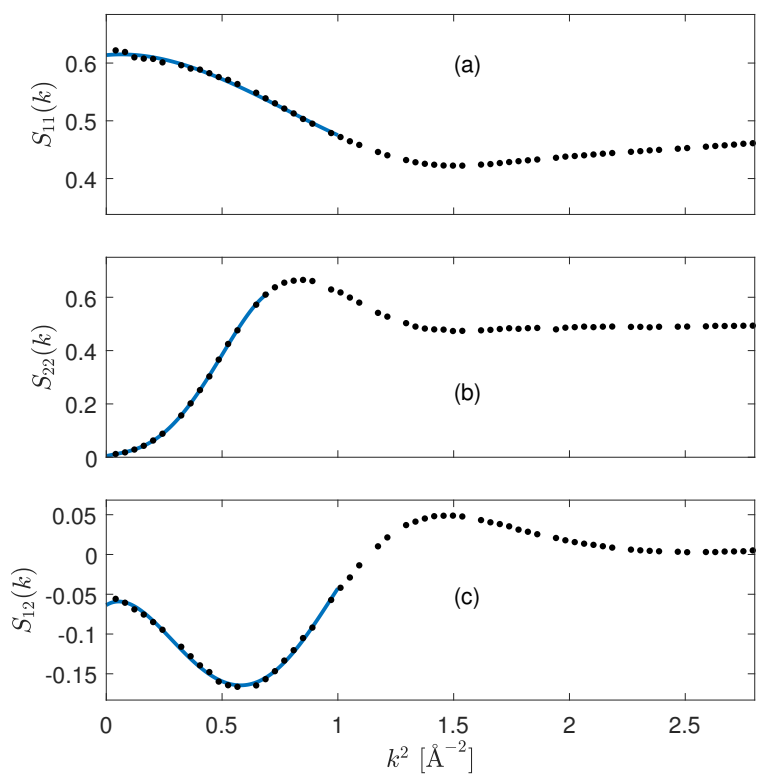

FIG. 4. Plot of the low-k values of the static structure factors of a system at a concentration of 95 wt.\% and temperature of $600 \mathrm{~K}$. A fourth-order polynomial line of best fit was used to obtain the $S_{\alpha \beta}(0)$ values.

the partial molecular volumes $\nu_{\alpha}$. The partial molecular volumes for trehalose have not been shown here, though they were found to have a qualitatively similar dependence on temperature and concentration to that seen in experimental systems [47]. The partial volume of the water molecule was found to be approximately $30 \AA^{3}$ as expected from experiment, while the trehalose molecule has a value of approximately $355 \AA^{3}$. The partial molecular volume decreases with temperature as the density of the fluid increases.

The thermodynamic factor is shown in Fig. 5 for (a) 2.2 wt.\% solution and (b) 95 wt.\% solution. This quantity decreases as the temperature decreases. The uncertainties also grow on the approach to $T_{g}$ as it becomes more difficult to get accurate values for $S_{\alpha \beta}(k \rightarrow$ $0)$. The thermodynamic factor enters into the equation for the mutual diffusion coefficient (eqn (8)) from the conversion between the true driving force of diffusion (chemical potential gradients $\left.\nabla \mu_{i}\right)$ to a quantity that can be easily measured (concentration gradients $\nabla n_{i}$ ). It is positive as the addition of a molecule of water adds chemical potential to the solution, but its decrease in magnitude at lower temperatures is the result of individual molecules having lower chemical potential. Therefore, this quantity acts to decrease the overall mutual diffusion coefficient at lower $T$, but not to the same dramatic extent as $L_{11}$. 

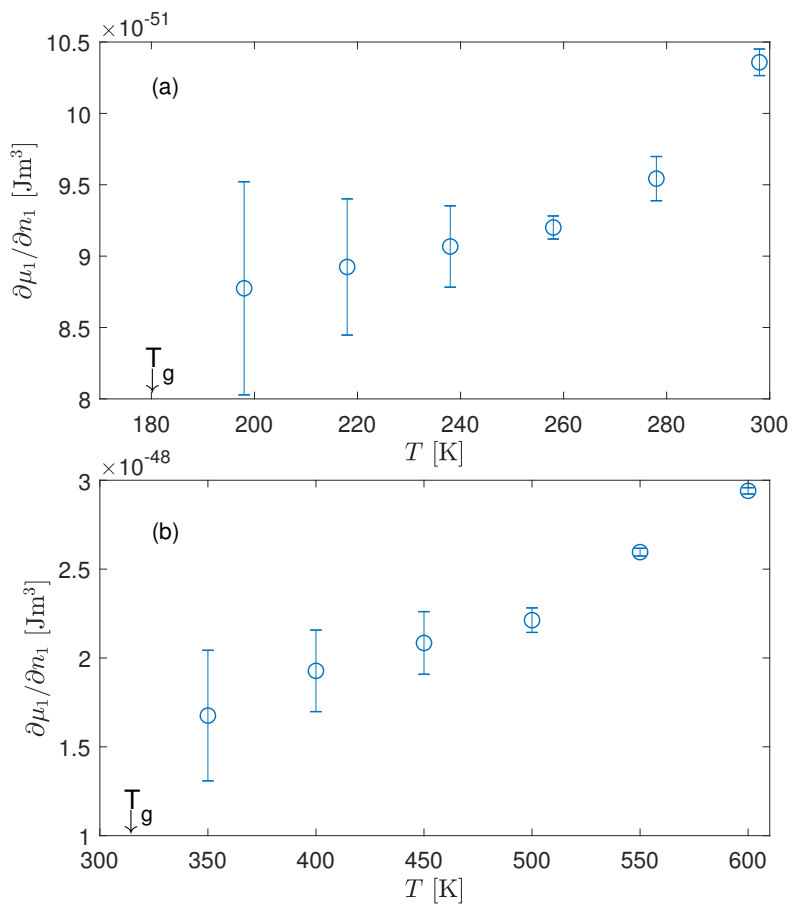

FIG. 5. Plot of the thermodynamic factor $\partial \mu_{1} / \partial n_{1}$ calculated from eqn (10) for (a) 2.2 wt.\% and (b) 95 wt.\% trehalose for temperatures approaching the corresponding glass transition temperature $T_{g}$. Note the $\mathrm{x}$ - and $\mathrm{y}$-axis scales differ.

The mutual diffusion coefficient was calculated from eqn (8) for all concentrations and temperatures. The mutual diffusion coefficients are shown in Fig. 6 for (a) 2.2 wt.\% and (b) 95 wt.\% solution, along with the glass transition temperature found for these concentrations. The mutual diffusion coefficient is observed to decrease on the approach to the glass transition temperature. The main quantities that contribute to the temperature dependence are the phenomenological coefficient $L_{11}$ and the thermodynamic factor $\partial \mu_{1} / \partial n_{1}$, both of which decrease on the approach to the glass transition. However, it is the $L_{11}$ coefficient decrease to zero at the glass transition temperature that causes $D_{m}$ to do the same.

To the authors' knowledge this is the first time that low temperature data for the mutual diffusion coefficient of trehalose has been presented from simulation or experiment. Literature data for the mutual diffusion coefficient of trehalose even at higher temperatures is sparse at best [26], which is surprising considering that trehalose is such an important cryoprotectant molecule [48]. Therefore, we are unable to check whether the values we calculate for $D_{m}$ quantitatively match with the experimental system on the approach to the 

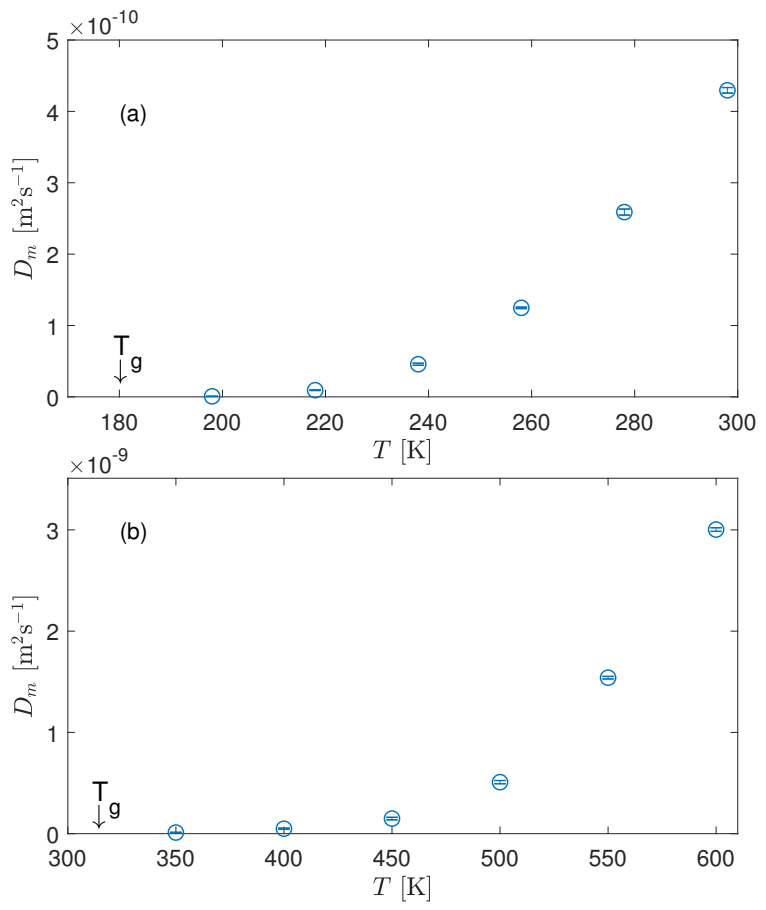

FIG. 6. Plot of the mutual diffusion coefficient $D_{m}$ calculated from eqn (8) for (a) 2.2 wt.\% and (b) 95 wt.\% trehalose for temperatures approaching the corresponding glass transition temperature $T_{g}$. Note the $\mathrm{x}$ - and $\mathrm{y}$-axis scales differ.

glass transition temperature. There is limited experimental data available for the similar molecule sucrose that shows reasonable quantitative agreement at $298 \mathrm{~K}$ [25], though no data at low temperatures are available. In any case, Fig. 6 shows that $D_{m}$ is observed to decrease on the approach to the glass transition at all concentrations studied, indicating that compositional relaxation slows as the temperature decreases. $D_{m}$ is also observed to decrease as the concentration increases, also indicating that the addition of more trehalose molecules slows compositional relaxation. However, it is difficult to directly compare $D_{m}$ at the same temperatures for different concentrations as trehalose is not completely soluble at all concentrations and temperatures.

Because of the limited accuracy with which the mutual diffusion coefficient can be calculated at low temperatures, it is difficult to determine if and where $D_{m}$ extrapolates to zero. The values at the lowest temperatures measured just above $T_{g}$ are found to be small but non-zero, though the trend in $D_{m}$ is consistent with a decline to a negligible value at $T_{g}$, exactly as observed in the colloidal suspension [18]. This trend is observed at all concentra- 


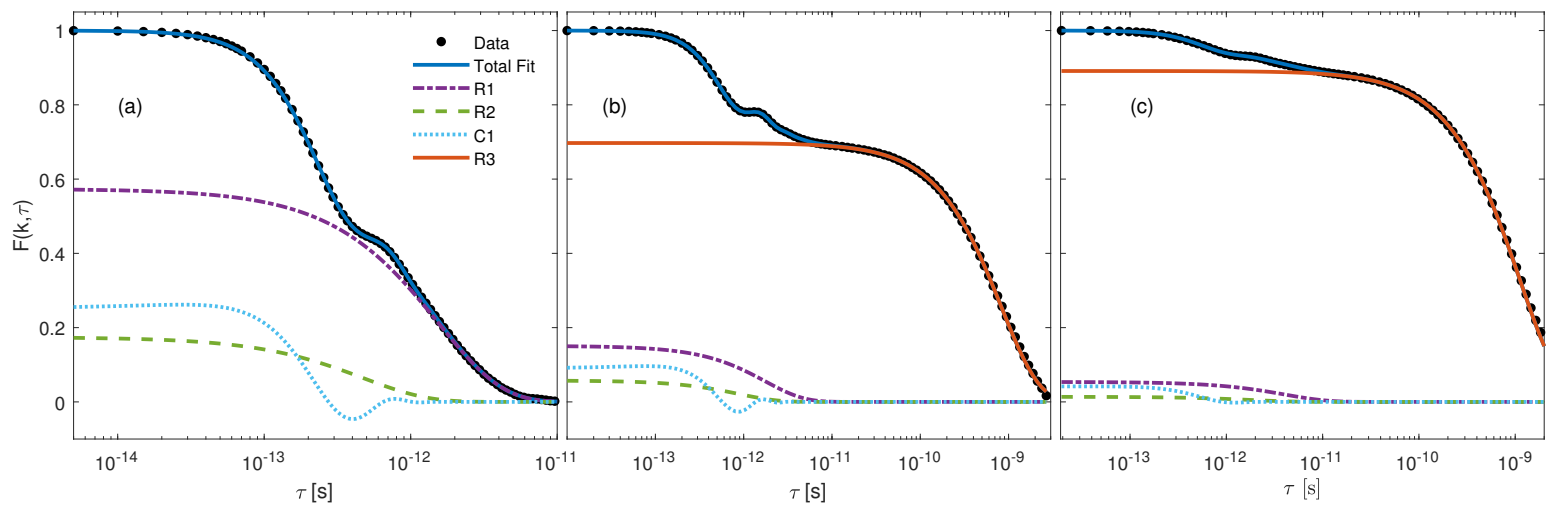

FIG. 7. Plot of the intermediate scattering function $F(k, \tau)$ of the water molecules at trehalose weight fractions of a) 0 wt.\%, b) 2.2 wt.\% and c) 9.0 wt.\% at a low wavevector of $0.3372 \AA^{-1}$ and temperature of $298 \mathrm{~K}$. Also shown is the total multiexponential fit and the various mode contributions.

tions studied, from the extremely low concentration shown in Fig. 6a, to the extremely high concentrations shown in Fig. $6 \mathrm{~b}$.

The decrease in $D_{m}$ determines the system's ability to make compositional changes, just as the $D_{-}$coefficient was in the colloidal suspension. Therefore, the decrease in $D_{m}$ to negligible values at $T_{g}$ shows that the glass transition correlates with the inability of the system to make the compositional changes needed to form a stable crystal.

\section{Intermediate Scattering Function}

In this section, results for the water molecules' intermediate scattering function $F(k, \tau)$ will be shown for low concentration solutions $(<10 \mathrm{wt} . \%)$ and extremely high concentration solutions $(>80 \mathrm{wt} . \%)$ at temperatures approaching the glass transition temperature $T_{g}$. The wave vectors studied were those consistent with the periodic boundaries of the simulation box, given by eqn (4) up to $a_{1}=a_{2}=a_{3}=10$, as our focus in this work is on the decay at low wavevectors close to the hydrodynamic limit. This is because it allows us to extrapolate to $k \rightarrow 0$ and compare the decay coefficients extracted from fits to the intermediate scattering function to the independently calculated mutual diffusion coefficients $D_{m}$.

$F(k, \tau)$ calculated for this system is shown in Fig. 7 for (a) pure water, (b) 2.2 wt.\% and (c) 9 wt.\% of trehalose. A multiexponential analysis was applied to the $F(k, \tau)$ data in 
order to isolate the individual mode contributions. The number of individual decay modes that need to be retained in eqn (14) to accurately fit the data depends on the state point and wavevector that is being studied. The data for the pure water required at most three real and one complex decay mode to accurately model the decay, owing to the complicated decay of $F(k, \tau)$ at short times. However, the addition of trehalose caused an extra long-time decay mode to appear, which we attribute to compositional relaxations.
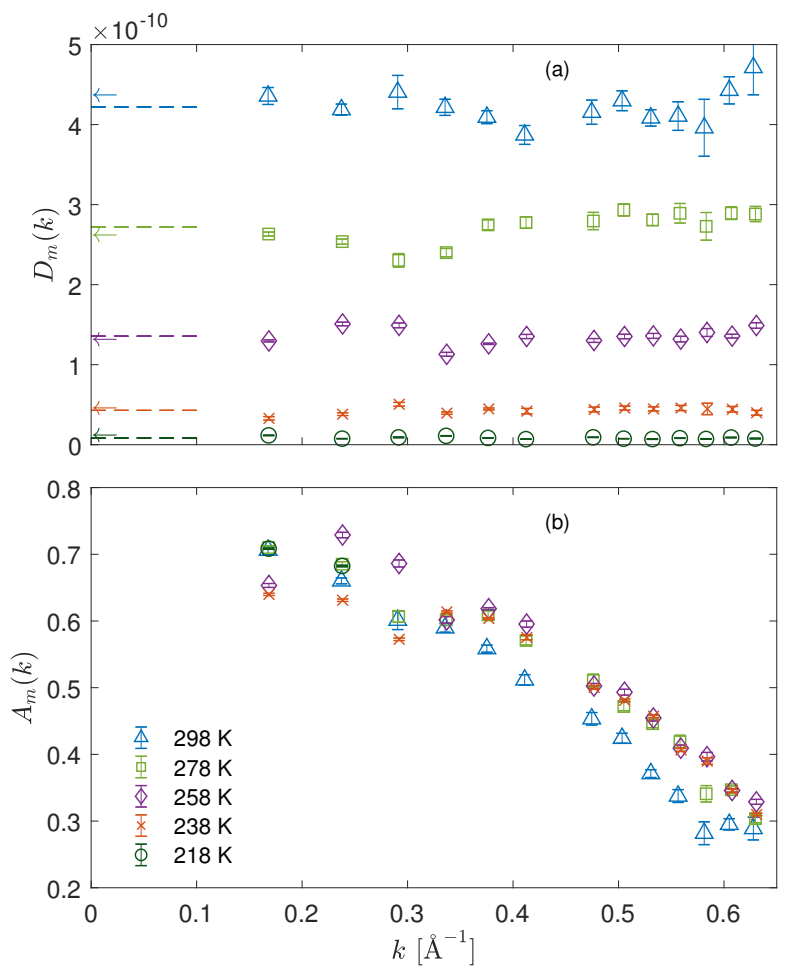

FIG. 8. Plot of wavevector dependence of the long time mode a) decay coefficients $D_{m}(k)$ and b) amplitudes $A_{m}(k)$. Data at trehalose concentration of 2.2 wt.\% and temperatures shown in the legend. Independently calculated values of $D_{m}$ from Fig. 6a are shown as arrows and dotted line shows average of $D_{m}(k)$ taken over the $k$ range shown.

The origin of the decay modes in the pure water system shown in Fig. 7a is not the focus of this work, and is only calculated to set a baseline for camparison after the addition of the trehalose. For a detailed discussion of a complete method to analyse collective correlation functions for water see the work by Omelyan and coworkers [49]. However, the decaying oscillatory function that results from the complex mode in the fit $(\mathrm{C} 1)$ could be related to the acoustic mode, and the real exponential terms $(R 1, R 2)$ to the heat mode, both of which are predicted from hydrodynamic theory [50] and have been observed in pure water 
systems $[51,52]$. The focus of this work is on the additional mode that emerges when trehalose molecules are added to the solution. In Fig. 7b we see that at the extremely low concentration of $2.2 \%$ the original short-time modes observed in pure water are still present, however their contribution to the complete decay of the intermediate scattering function has greatly diminished. These short-time modes now only make up $\approx 40 \%$ of the complete decay at 2.2 wt.\%, and only $\approx 10 \%$ of the decay at 9 wt. $\%$, with this trend continuing at higher concentrations. At moderate concentrations the decay is dominated by the new long-time relaxation mode that is not present in the pure water system. This shows that the intermediate scattering function in the sugar solution does not decay via short-time dynamics, but via a different long-time mechanism.

At just 2.2 wt.\%, this new mode extends the relaxation of the intermediate scattering function out by over two orders of magnitude, showing a significant slow-down in the relaxation of the Fourier components of the water molecule number density. This would have the effect of inhibiting the crystallisation of the water as the compositional changes needed to meet the stoichiometry of a crystal containing trehalose and water would occur very slowly. This crystallisation inhibition is observed experimentally through the large effect that the solute has on lowering the homogenous nucleation temperature $T_{h}$ of the water, which is the lowest temperature that supercooled water can achieve using experimental cooling rates before crystallisation occurs. However, it is interesting to note that even though the dynamics of the water molecules has slowed down, it is not enough to noticeably raise the glass transition temperature at these cooling rates. As we saw in Fig. 2, large concentrations of trehalose are needed to increase the relaxation time by enough to result in a significant increase in $T_{g}$ at these ultra fast cooling rates.

It has been shown in ionic liquids [27], liquids containing dissolved gases [53, 54], binary Lennard-Jones fluids [28] and model colloidal suspensions [17, 18] that the slowest decay mode observed in the intermediate scattering function is related to interdiffusion coefficients. It is conceivable that the slowest decay mode in this sugar solution may be a different process that is not related to collective diffusion, so here we seek to determine the source of the long-time mode in the intermediate scattering function. To do this we extracted the decay-coefficients of the long-time mode from the fits to compare against the independently calculated $D_{m}$ values given in Fig. 6a. The extracted decay coefficients are shown in Fig. 8a (symbols) along with the independently calculated mutual diffusion coefficients (arrows). 
The decay coefficient seems to be wavevector independent, so in order to determine the $k \rightarrow 0$ value an average was done over the wavevector range shown, and is indicated by the dotted line in Fig. 8a. As can be observed, in the $k \rightarrow 0$ limit the decay coefficients of the long-time decay of the intermediate scattering function are in good agreement with the independently measured mutual diffusion coefficients, as was found in the colloidal system [18]. This can be shown at all concentrations provided that the long-time exponential decay mode can be isolated. This becomes harder at higher concentrations or lower temperatures, as wave vector needed to achieve the macroscopic diffusive limit (where we see exponential decay) decreases, so larger simulation sizes are needed ( $\left.\operatorname{since} k_{\min }=2 \pi / L\right)$. This is evidence that the slowest collective process limiting the decay of the intermediate scattering function is also compositional relaxation.

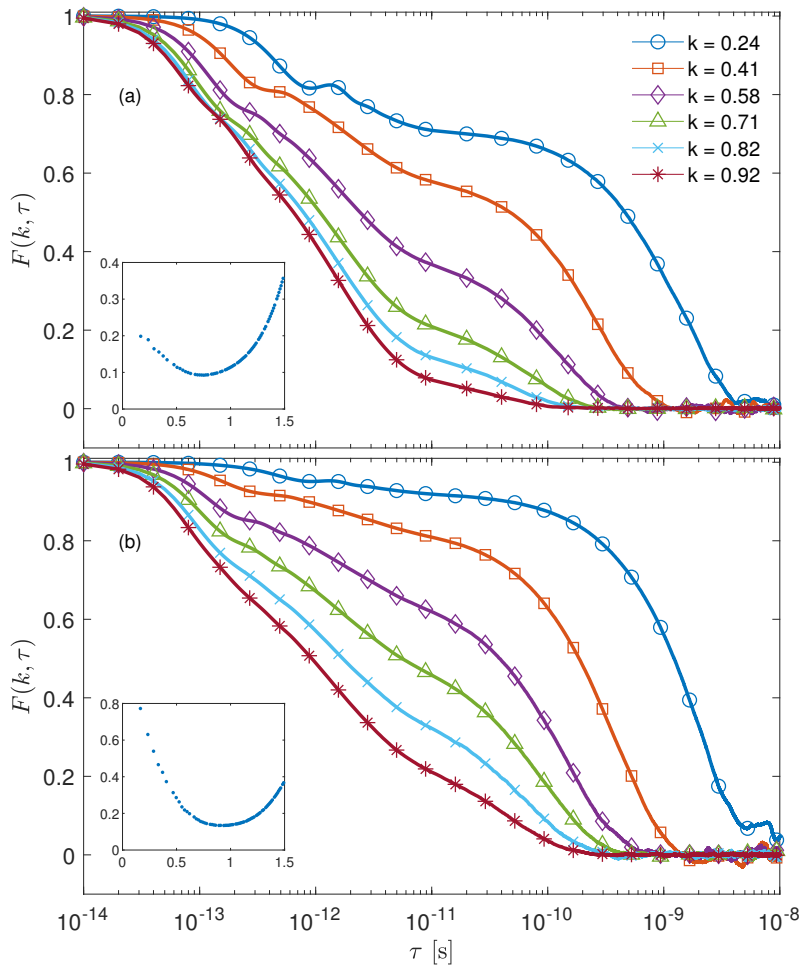

FIG. 9. Plot of the water intermediate scattering function $F(k, \tau)$ at trehalose weight fractions of a) 2.2 wt.\% and b) 9 wt.\% at $298 \mathrm{~K}$ for wavevectors $k$ shown in the legend. Inset shows $S(k)$ at low wavevectors to help visualise the position of the wavevectors in the structure factor.

The amplitudes $A_{m}(k)$ were also extracted from the fits to the long-time mode and they are shown in Fig. 8b. The compositional mode amplitudes have a maximum in the 
$k \rightarrow 0$ limit that corresponds to long wavelength density fluctuations. As $k$ increases, shorter wavelength density fluctuations are being probed, so the compositional relaxation mode has a smaller contribution. At moderate wavevectors of $k>0.7-0.8 \AA^{-1}$, the amplitude of this mode decreases to where it makes an insignificant contribution to the decay of the intermediate scattering function. This type of behaviour was also observed in the colloidal suspension [18] and we explained this by saying that high- $k$ density fluctuations would decay via small-scale local movements, and would not need large scale compositional rearrangements to decay.

To better visualise the wavevector dependence of the compositional relaxation mode we have plotted the intermediate scattering function in Fig. 9 at a number of low wavevectors for a) 2.2 wt.\% and b) 9.0 wt.\% trehalose. The insets show the values of the structure factor $S(k)$ to help us visualise the wavevectors being probed. The decay rate of $F(k, \tau)$ is slowest at the lowest $k$ where long wavelength density fluctuations are being probed, and it increases with increasing $k$ as shorter wavelength density fluctuations decay faster. As we saw previously, the $k$ dependence of $D_{m}(k)$ is (roughly) independent of wavevector, so the decay rate of $F(k, \tau)$ scales with $k^{2}$ as expected. This mode can be seen by the secondary hump in the decay, and we see that its amplitude is maximum in the low- $k$ limit, and decreases as $k$ increases. At $k \approx 1.0 \AA^{-1}$ the mode amplitude is quite small, showing that this process is only significant for the decay of large wavelength density fluctuations. However as the concentration of trehalose increases, this mode persists to larger $k$. This can be seen be comparing the decay at $k=0.92 \AA^{-1}$ at both concentrations where the amplitude is larger for the 9 wt.\% solution.

Since the long-time decay of the intermediate scattering function is governed by the mutual diffusion coefficient, and this coefficient decreases on the approach to the glass transition, we expect the long-time decay of the intermediate scattering function to slow down on the approach to $T_{g}$ and eventually plateau at $T_{g}$ as $D_{m} \rightarrow 0$. Fig. 10 shows the decay of the intermediate scattering function for a) $2.2 \mathrm{wt} . \%$ and b) $95 \mathrm{wt} . \%$ at temperatures down to and just beyond $T_{g}$ and wavevector of $0.33 \AA^{-1}$. We have chosen to focus on these two concentrations as they allow us to show the two extremes in the behaviour of the intermediate scattering function. Solutions at these concentrations remain single-phase at temperatures close to their glass transition temperatures, allowing us to obtain data at each state point. We note that no ageing was observed for systems with temperatures above $T_{g}$. 


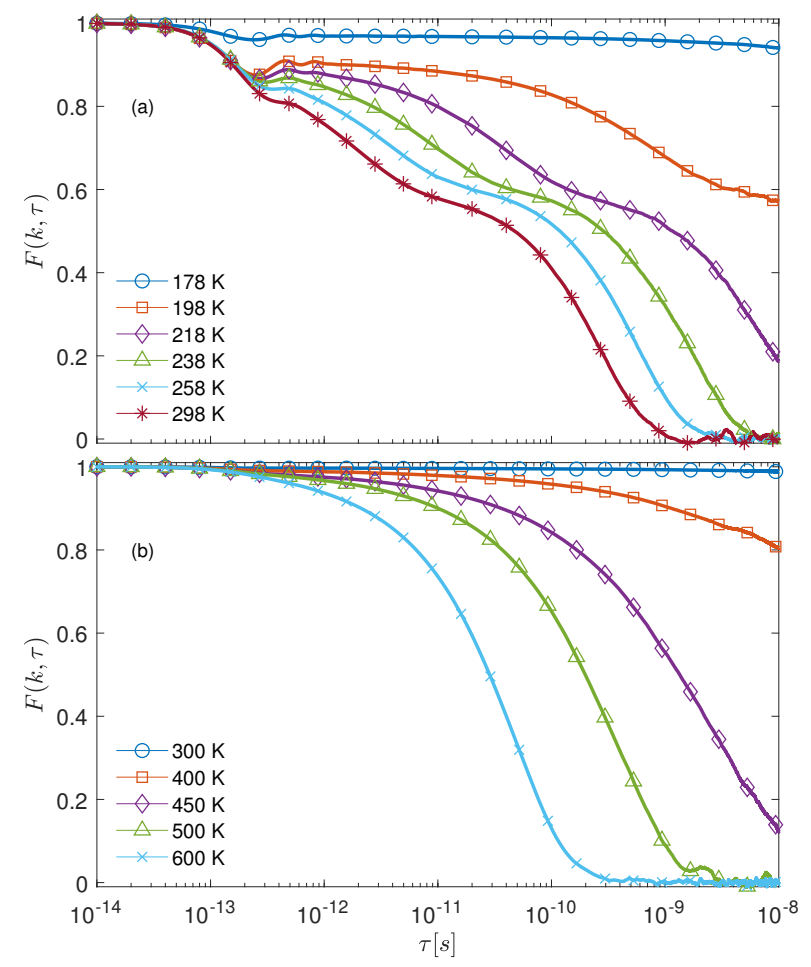

FIG. 10. Plot of the water intermediate scattering function $F(k, \tau)$ at trehalose weight fractions of a) 2.2 wt. $\%$ and b) 95.0 wt. $\%$ at $k=0.33 \AA^{-1}$ for temperatures leading down to the glass transition.

The $F(k, \tau)$ decay at 2.2 wt.\% shown in Fig. 10a highlights two key processes as the temperature decreases. First, the acoustic mode seen by the reversal in $F(k, \tau)$ at $\approx 4 \times 10^{-13}$ $\mathrm{s}$ is decaying slower as the solution becomes more solid like and the soundwave is able to propagate further as it is not being dissipated in the solution. Second, the long-time compositional mode decay, which is the limiting factor in the decay of the intermediate scattering function, decays more slowly. At temperatures below the glass transition temperature, both the sound mode and the mutual diffusion mode no longer decay on a reasonable timescale, and so we see the classic plateau in the intermediate scattering function which signifies that a glass has formed.

The decay at 95.0 wt.\% is simpler. It shows a continuation of the trend already observed in Fig. 7 at low concentrations. The addition of trehalose suppresses the short time dynamic relaxation so that the intermediate scattering function decays completely via long-time compositional relaxation. The decay rate of this mode decreases as the temperature decreases, until the relaxation time increases to well beyond the maximum delay time of the corre- 
lation function, and the system has formed a glass. At both concentrations the long-time decay rate decreases on the approach to the glass transition temperature. This results in an extremely slow decay at temperatures slightly below $T_{g}$. This shows that over the timescale observable in our MD simulations, the slowing down and eventual 'arrest' that is observed in the long-time tail of the intermediate scattering function is directly linked to the slow-down and arrest in compositional relaxations.

\section{CONCLUSION}

Molecular dynamics simulations were conducted on a model trehalose solution to study its behaviour on its approach to the glass transition. In this study, we sought to determine whether the arrest of compositional relaxations could be linked to the glass transition of the solution, a link which has been observed in model colloidal suspensions. We first determined the glass transition temperature of the solution at various concentrations by quenching the fluid and calculating the constant pressure heat capacity. A sudden inflection in the heat capacity allowed us to determine $T_{g}$. We found that small concentrations of trehalose $(<9$ wt.\%) did not increase $T_{g}$ by a measurable amount, but very large concentrations $>80$ wt. $\%$ had a large effect on $T_{g}$.

The mutual diffusion coefficients were calculated from equilibrium MD simulations using a combination of Green-Kubo and Kirkwood-Buff theory. This was done for a number of temperatures and concentrations, which is the first time that the mutual diffusion coefficient (and all the factors that contribute) has been determined for concentrated trehalose solutions in simulation or experiment. $D_{m}$ was found to decrease with increased trehalose concentration. It also decreased with temperature showing that composition is relaxing more slowly at lower temperatures. This culminated in a decrease to negligible values at $T_{g}$, something that has also been observed in colloidal suspensions. This demonstrates that these two very different glass forming systems have something in common: under conditions where the composition cannot relax, they are not able to reach their equilibrium structure within the simulation time window.

The mutual diffusion coefficient was shown to correspond to the slowest decay mode in the intermediate scattering function at low wavevectors. Therefore, the decrease in $D_{m}$ to negligible values resulted in an extremely slow decay in the intermediate scattering func- 
tion and the formation of a plateau, which is usually associated with the arrest and glass formation. This slow decay mode was found to become more dominant as the concentration increases, showing that the relaxation of the intermediate scattering function at large concentrations is dominated by the relaxation of composition.

This work shows the importance of the mutual diffusion coefficient and the relaxation of composition to the process of vitrification. While the self-diffusion coefficient and the viscosity both decrease as solidification is approached for both good and poor glass forming solutions, this is not necessarily the case for the mutual diffusion coefficient, which can increase with concentration for some binary solutions as they approach solidification. By observing the strong decrease in the mutual diffusion coefficient as the glass transition is approached in two very different good glass formers (polydisperse colloidal suspensions and trehalose solutions) we have identified behaviour that contrasts with the increase in the mutual diffusion coefficient seen previously in a crystallising fluid (the monodisperse colloidal suspension). This suggests that the mutual diffusion coefficient, which is far less commonly studied than the self diffusion coefficient or the viscosity, may be useful in discriminating between good and poor glass formers. We believe it would be prudent to obtain more ex-

perimental and simulation data on the mutual diffusion coefficient in trehalose solutions as this is currently lacking. More experimental and simulation studies looking at this relationship in other glass forming systems would also be beneficial to test the generality of the observations made.

\section{ACKNOWLEDGMENTS}

Computational resources were provided by the National Computation Infrastructure (NCI). S.D.W.H. acknowledges the Australian government for supporting this work through an Australian post-graduate award (APA). G.B. acknowledges the support of the Australian Government through the Australian Research Council's Linkage Projects funding scheme (project LP140100993).

[1] Z. Hubálek, Cryobiology 46, 205 (2003). 
[2] A. Kaczmarczyk, B. Funnekotter, S. R. Turner, E. Bunn, G. Bryant, T. E. Hunt, and R. L. Mancera, CryoLetters 34, 508 (2013).

[3] G. Bryant, Cryobiology 32, 114 (1995).

[4] J. Wolfe and G. Bryant, Cryobiology 39, 103 (1999).

[5] V. Berejnov, N. S. Husseini, O. A. Alsaied, and R. E. Thorne, Journal of Applied Crystallography 39, 244 (2006), arXiv:0604109 [physics]; S. N. Bhat, A. Sharma, and S. V. Bhat, Physical Review Letters 95, 235702 (2005); P. Brüggeller and E. Mayer, Nature 288, 569 (1980); G. M. Fahy, B. Wowk, J. Wu, and S. Paynter, Cryobiology 48, 22 (2004).

[6] L. M. Crowe, D. S. Reid, and J. H. Crowe, Biophysical Journal 71, 2087 (1996).

[7] J. H. Crowe, J. F. Carpenter, and L. M. Crowe, Annual Review of Physiology 60, 73 (1998); K. L. Koster and A. C. Leopold, Plant physiology 88, 829 (1988); K. L. Koster, ibid. 96, 302 (1991).

[8] J. H. Crowe, L. M. Crowe, A. E. Oliver, N. Tsvetkova, W. Wolkers, and F. Tablin, Cryobiology 43, 89 (2001); G. Bryant, K. L. Koster, and J. Wolfe, Seed science research 11, 17 (2001); P. Westh, Physical Chemistry Chemical Physics 10, 4110 (2008); H. D. Andersen, C. Wang, L. Arleth, G. H. Peters, and P. Westh, Proceedings of the National Academy of Sciences 108, 1874 (2011).

[9] B. Kent, T. Hauß, B. Demé, V. Cristiglio, T. Darwish, T. Hunt, G. Bryant, and C. J. Garvey, Langmuir 31, 9134 (2015).

[10] B. Kent, T. Hunt, T. A. Darwish, T. Hauß, C. J. Garvey, and G. Bryant, Journal of The Royal Society Interface 11, 20140069 (2014).

[11] T. Lenné, C. J. Garvey, K. L. Koster, and G. Bryant, The Journal of Physical Chemistry. B 113, $2486(2009)$.

[12] K. L. Koster, K. J. Maddocks, and G. Bryant, European biophysics journal 32, 96 (2003).

[13] K. L. Koster, Y. P. Lei, M. Anderson, S. Martin, and G. Bryant, Biophysical journal 78, 1932 (2000).

[14] G. M. Fahy, B. Wowk, J. Wu, J. Phan, C. Rasch, A. Chang, and E. Zendejas, Cryobiology 48, 157 (2004).

[15] F. Sussich, R. Urbani, F. Princivalle, and A. Cesàro, Journal of the American Chemical Society 120, 7893 (1998). 
[16] P. B. Conrad and J. J. de Pablo, Journal of Physical Chemistry A 103, 4049 (1999); L. Weng and G. D. Elliott, Physical Chemistry Chemical Physics 16, 11555 (2014), arXiv:15334406; N. Ekdawi-Sever, J. J. de Pablo, E. Feick, and E. von Meerwall, Journal of Physical Chemistry A 107, 936 (2003).

[17] S. D. Hannam, P. J. Daivis, and G. Bryant, Physical Review E 94, 012619 (2016).

[18] S. D. W. Hannam, P. J. Daivis, and G. Bryant, Physical Review E 96, 022609 (2017).

[19] B. Bernu, Y. Hiwatari, and J. P. Hansen, J. Phys. C: Solid State Phys. 18, L371 (1985).

[20] U. K. Nandi, A. Banerjee, S. Chakrabarty, and S. M. Bhattacharyya, J. Chem. Phys. 145, 034503 (2016).

[21] U. R. Pedersen, T. B. Schrøder, and J. C. Dyre, Phys. Rev. Lett. 120, 165501 (2018).

[22] J. Berry and M. Grant, Phys. Rev. E 89, 062303 (2014).

[23] P. Mazur, The American Journal of Physiology 247, C125 (1984).

[24] T. Sei, T. Gonda, and Y. Arima, Journal of Crystal Growth 240, 218 (2002); T. Gonda and T. Sei, Progress in Crystal Growth and Characterization of Materials 51, 70 (2005).

[25] A. C. Ribeiro, O. Ortona, S. M. Simões, C. I. Santos, P. M. Prazeres, A. J. Valente, V. M. Lobo, and H. D. Burrows, Journal of Chemical and Engineering Data 51, 1836 (2006).

[26] X. He, A. Fowler, and M. Toner, Journal of Applied Physics 100, 074702 (2006).

[27] M. H. Rausch, J. Lehmann, A. Leipertz, and A. P. Fröba, Physical Chemistry Chemical Physics 13, 9525 (2011).

[28] J. W. Nichols and D. R. Wheeler, Industrial and Engineering Chemistry Research 54, 12156 (2015).

[29] M. S. Green, The Journal of Chemical Physics 22, 398 (1954).

[30] R. Kubo, Journal of the Physical Society of Japan 12, 570 (1957).

[31] J. G. Kirkwood and F. P. Buff, The Journal of Chemical Physics 19, 774 (1951).

[32] S. R. de Groot and P. Mazur, Non-equilibrium thermodynamics (Dover Publications, New York, 1984).

[33] F. Barocchi, U. Bafile, and M. Sampoli, Physical Review E 85, 022102 (2012).

[34] W. L. Jorgensen, J. D. Madura, and C. J. Swenson, Journal of the American Chemical Society 106, $6638(1984)$.

[35] W. Damm, A. Frontera, J. Tirado-Rives, and W. L. Jorgensen, Journal of Computational Chemistry 18, 1955 (1997). 
[36] W. L. Jorgensen, D. S. Maxwell, and J. Tirado-Rives, Journal of the American Chemical Society 118, 11225 (1996).

[37] H. J. C. Berendsen, J. R. Grigera, and T. P. Straatsma, The Journal of Physical Chemistry 91, 6269 (1987).

[38] C. A. Kreck and R. L. Mancera, Journal of Physical Chemistry B 118, 1867 (2014).

[39] S. Plimpton, Journal of Computational Physics 117, 1 (1995).

[40] M. E. Tuckerman, J. Alejandre, R. López-Rendón, A. L. Jochim, and G. J. Martyna, Journal of Physics A: Mathematical and General 39, 5629 (2006).

[41] C. A. Angell, Chemical Reviews 102, 2627 (2002).

[42] C. A. Angell and S. Borick, Journal of Non-Crystalline Solids 307-310, 393 (2002), arXiv:0202304 [cond-mat].

[43] A. Hallbrucker, E. Mayer, and G. P. Johari, The Journal of Physical Chemistry 93, 4986 (1989).

[44] V. Velikov, S. Borick, and A. C. A., Science 294, 2335 (2001).

[45] Y. Yue and C. A. Angell, Nature 427, 717 (2004).

[46] T. Chen, A. Fowler, and M. Toner, Cryobiology 40, 277 (2000).

[47] C. Branca, S. Magazù, G. Maisano, and P. Migliardo, Journal of Biological Physics 26, 295 (2000).

[48] H. Huang, G. Zhao, Y. Zhang, J. Xu, T. L. Toth, and X. He, ACS Biomaterials Science and Engineering 3, 1758 (2017).

[49] I. P. Omelyan, I. M. Mryglod, and M. V. Tokarchuk, Condensed Matter Physics 8, 25 (2005).

[50] J. P. Boon and S. Yip, Molecular hydrodynamics (Dover Publications, New York, 2013).

[51] T. Komatsu, N. Yoshii, S. Miura, and S. Okazaki, Fluid Phase Equilibria 226, 345 (2004).

[52] C. Y. Liao, S. H. Chen, and F. Sette, Physical Review E 61, 1518 (2000).

[53] A. Heller, T. M. Koller, M. H. Rausch, M. S. H. Fleys, A. N. R. Bos, G. P. van der Laan, Z. A. Makrodimitri, I. G. Economou, and A. P. Fröba, The Journal of Physical Chemistry B 118, 3981 (2014).

[54] A. Heller, C. Giraudet, Z. A. Makrodimitri, M. S. Fleys, J. Chen, G. P. Van Der Laan, I. G. Economou, M. H. Rausch, and A. P. Fröba, Journal of Physical Chemistry B 120, 10808 (2016). 